\title{
Acupuncture and Auricular Acupressure in Relieving Menopausal Hot Flashes of Bilaterally Ovariectomized Chinese Women: A Randomized Controlled Trial
}

\author{
Jue Zhou, ${ }^{1}$ Fan Qu, ${ }^{2}$ Xisheng Sang, ${ }^{3}$ Xiaotong Wang, ${ }^{4}$ and Rui Nan ${ }^{5}$ \\ ${ }^{1}$ Sino-Britain Joint Laboratory, College of Pharmaceutical Sciences, Zhejiang University, Hangzhou, Zhejiang 310058, China \\ ${ }^{2}$ Women's Hospital, School of Medicine, Zhejiang University, Hangzhou, Zhejiang 310006, China \\ ${ }^{3}$ Heilongjiang University of Chinese Medicine, Harbin, Heilongjiang 150040, China \\ ${ }^{4}$ The First Affiliated Hospital, Liaoning University of Chinese Medicine, Shenyang, Liaoning 110032, China \\ ${ }^{5}$ Colorado School of Traditional Chinese Medicine, 1441 York Street, Denver, CO 80206, USA
}

Correspondence should be addressed to Fan Qu, qufan43@yahoo.com.cn

Received 8 May 2008; Accepted 12 January 2009

Copyright (C) 2011 Jue Zhou et al. This is an open access article distributed under the Creative Commons Attribution License, which permits unrestricted use, distribution, and reproduction in any medium, provided the original work is properly cited.

\begin{abstract}
The objective of this study is to explore the effects of acupuncture and auricular acupressure in relieving menopausal hot flashes of bilaterally ovariectomized Chinese women. Between May 2006 and March 2008, 46 bilaterally ovariectomized Chinese women were randomized into an acupuncture and auricular acupressure group $(n=21)$ and a hormone replacement therapy (HRT) group (Tibolone, $n=25$ ). Each patient was given a standard daily log and was required to record the frequency and severity of hot flashes and side effects of the treatment felt daily, from 1 week before the treatment started to the fourth week after the treatment ended. The serum levels of follicle stimulating hormone (FSH), LH and $\mathrm{E}_{2}$ were detected before and after the treatment. After the treatment and the follow-up, both the severity and frequency of hot flashes in the two groups were relieved significantly when compared with pre-treatment $(P<.05)$. There was no significant difference in the severity of hot flashes between them after treatment $(P>.05)$, while after the follow-up, the severity of hot flashes in the HRT group was alleviated more. After the treatment and the follow-up, the frequency of menopausal hot flashes in the HRT group was reduced more $(P<.05)$. After treatment, the levels of FSH decreased significantly and the levels of $\mathrm{E}_{2}$ increased significantly in both groups $(P<.05)$, and they changed more in the HRT group $(P<.05)$. Acupuncture and auricular acupressure can be used as alternative treatments to relieve menopausal hot flashes for those bilaterally ovariectomized women who are unable or unwilling to receive HRT.
\end{abstract}

\section{Introduction}

Hot flashes occur in the vast majority of post-menopausal women [1]. An extensive questionnaire study of 506 women found that $87 \%$ had daily hot flashes [2]. Hot flashes are episodic and usually accompanied by nausea, dizziness, headache, palpitations, diaphoresis or night sweats [3]. Having hot flashes may decrease a woman's quality of life by decreasing the quality of sleep and aggravating fatigue and depression $[4,5]$. Menopausal hot flashes make most of women seek medical care during the menopausal transition [2]. Menopausal hot flashes are related to a psychological or mental disorder in menopausal women and the hormonal changes in these women may be the underlying mechanism $[6,7]$.
For those bilaterally ovariectomized pre-menopausal women, the estradiol $\left(\mathrm{E}_{2}\right)$ contents in their serum were reduced by $80 \%$ [8]. The abrupt decline in $\mathrm{E}_{2}$ usually leads to more frequent and severe menopausal symptoms, especially hot flashes. Menopausal hot flashes are also related to enhanced norepinephrine (NE) activity in the hypothalamus, resulting in an abrupt, transient, downward resetting of the normal thermoregulatory response set point $[9,10]$. Most of the bilaterally ovariectomized Chinese women have difficulty in stopping hormone replacement therapy (HRT) due to the severe menopausal symptoms. Although HRT historically has been used as the standard treatment for hot flashes [11], many women choose not to initiate or adhere to HRT because of its potential health risks and side effects $[12,13]$. In recent years, non-pharmacological alternative 
treatments are being requested by more bilaterally ovariectomized women to relieve their menopausal symptoms, especially menopausal hot flashes. A study has demonstrated that acupuncture could induce accumulation of vaginal exfoliative cells, increase the weight of adrenal, and raise the level of serum corticosterone in ovariectomized model rats [14]. It is deduced that estrogen levels may be elevated using acupuncture to create compensatory hyperplasia of the adrenal cortex, thereby enhancing the transferring of androgen into estrogen in peripheral tissues [14]. In as early as 1995, the acupuncture's efficacy in relieving menopausal symptoms has been demonstrated [15]. Alternative and complementary therapies, including acupuncture, have been used increasingly in recent years to relieve menopausal symptoms [16-23], although one of them suggested that the used medical acupuncture was not any more effective for reducing menopausal hot flashes than was the chosen sham acupuncture [16]. As hot flashes are the most common menopausal symptoms in bilaterally ovariectomized women, it is important to find effective, non-pharmacological treatments to relieve their menopausal hot flashes. This study was designed to explore the effects of acupuncture and auricular acupressure in relieving menopausal hot flashes of bilaterally ovariectomized Chinese women.

\section{Subjects and Methods}

2.1. Subjects. Between May 2006 and March 2008, 46 bilaterally ovariectomized Chinese women were recruited through advertisement to complete 12 weeks of intervention with either acupuncture and auricular acupressure or Livial (Tibolone).

Inclusion criteria were as follows: the subject had received a bilateral ovariectomy in the previous 2 years and suffered from menopausal hot flashes; the patient did not manifest any perimenopausal symptoms before the bilateral ovariectomy and had not taken any drugs containing hormones or affecting the cardiovascular system during the previous 6 months; the level of thyroid-stimulating hormone (TSH) was normal and the $\mathrm{E}_{2}$ concentration was $<50 \mathrm{pg} / \mathrm{mL}$; a gynecologic examination and laboratory tests showed that the patient did not suffer from other organic diseases of the reproductive system after the bilateral ovariectomy; written consent was obtained from the subject stating that the subject would complete the study. To have been considered for the study, patients must have met all criteria.

Exclusion criteria were as follows: the subject was under other medical treatment during the research period, had metabolic, renal, anaphylactic or endocrine disease, or suffered from primary hypertension, primary hypotension, chronic anemia, tuberculosis, a mental disorder or a chronic affection; the body mass index (BMI) of the subject was more than 24 or she was a cigarette smoker. Patients were excluded from the study if they fit any of the above criteria.

The women were informed of the short- and long-term benefits of HRT and were informed about the aim and methodology of the study. Ethical approval and permission to conduct the study were obtained from the local ethical committee and the administration of the study was based on

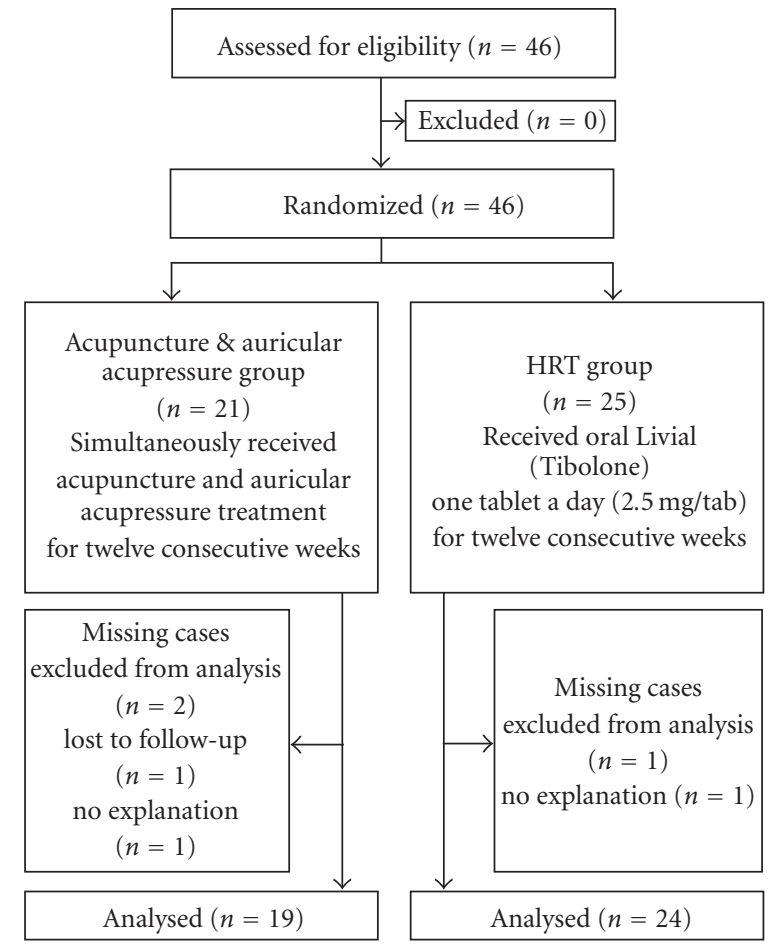

FIgURe 1: Study design.

international ethical guidelines. Voluntary participation was requested and informed consent was obtained.

Subjects were randomized to either the acupuncture and auricular acupressure group or the HRT group with the use of a randomization chart constructed in Microsoft Excel that randomized numbers into two groups. Having been divided, the acupuncture and auricular acupressure group had 21 cases and HRT group had 25. In the statistical analysis, 43 of the women were included. Three subjects were considered missing cases during the study (Figure 1). There was no significant difference in baseline characteristics between the two groups (Table 1).

Each patient was given a standard daily log and was required to record the frequency and severity of hot flashes and side effects of the treatment felt daily, from 1 week before the treatment started to the fourth week after the treatment ended. The patients were required to record the items before going to bed in the evening. The standard daily log was made in a structured way by the hospitals and all the possible side effects of the treatment had been listed on it, which had been validated beforehand.

Each participant received a physical examination, a routine blood examination, a routine uronoscopy, a liver function test and a renal function test, respectively, 1 day before the treatment started and 1 day after the treatment ended.

\subsection{Group and Administration}

2.2.1. Acupuncture and Auricular Acupressure Group. Each patient randomized into the acupuncture and auricular 
TABLE 1: The baseline characteristics of the participants.

\begin{tabular}{|c|c|c|}
\hline Item & Acupuncture and auricular acupressure group $(n=19)$ & HRT group $(n=24)$ \\
\hline \multicolumn{3}{|l|}{ Demographic characteristics } \\
\hline Age (years) & $41.6 \pm 5.8$ & $42.8 \pm 4.1$ \\
\hline Time since ovariectomy (years) & $1.3 \pm 0.4$ & $1.2 \pm 0.5$ \\
\hline Married/living as married & $14(73.7 \%)$ & $18(75.0 \%)$ \\
\hline Divorced/separated & $4(21.1 \%)$ & $5(20.8 \%)$ \\
\hline Never married & $1(5.3 \%)$ & $1(4.2 \%)$ \\
\hline Women with children & $14(73.7 \%)$ & $14(58.3 \%)$ \\
\hline \multicolumn{3}{|l|}{ Area of residence } \\
\hline Urban & $12(63.2 \%)$ & $21(87.5 \%)$ \\
\hline Suburban & $3(15.8 \%)$ & $2(8.3 \%)$ \\
\hline Small town & $2(10.5 \%)$ & 0 \\
\hline Rural & $2(10.5 \%)$ & $1(4.2 \%)$ \\
\hline \multicolumn{3}{|l|}{ Current employment status } \\
\hline Full-time employment & $8(42.1 \%)$ & $18(75.0 \%)$ \\
\hline Part-time employment & $5(26.3 \%)$ & $2(8.3 \%)$ \\
\hline Registered unemployed & $3(15.8 \%)$ & $2(8.3 \%)$ \\
\hline Retired & $3(15.8 \%)$ & $2(8.3 \%)$ \\
\hline \multicolumn{3}{|l|}{ Cause of ovariectomy } \\
\hline Bilateral tubal-ovarian cyst & $2(10.5 \%)$ & $8(33.3 \%)$ \\
\hline Bilateral tubal-ovarian abscess & $3(15.8 \%)$ & $2(8.3 \%)$ \\
\hline Malignant tumor of uterus and/or ovary & $3(15.8 \%)$ & $2(8.3 \%)$ \\
\hline Benign tumor of uterus and/or ovary & $2(10.5 \%)$ & $3(12.5 \%)$ \\
\hline Removing the need for contraception & $1(5.3 \%)$ & $2(8.3 \%)$ \\
\hline Prophylactic ovariectomy & $2(10.5 \%)$ & $1(4.2 \%)$ \\
\hline Bilateral severe ovarian endometriosis & $3(15.8 \%)$ & $2(8.3 \%)$ \\
\hline Cessation of menstruation & $2(10.5 \%)$ & $2(8.3 \%)$ \\
\hline Other & $1(5.3 \%)$ & $2(8.3 \%)$ \\
\hline Duration of the menopausal hot flashes (year) & $1.1 \pm 0.5$ & $1.3 \pm 0.4$ \\
\hline
\end{tabular}

acupressure group received both acupuncture and auricular acupressure treatments.

Acupuncture. The selected acupoints: Sanyinjiao (SP6), Fengchi (GB20), Hegu (LI4), Quchi (LI11), Guanyuan (CV4), Dazhui (GV14), Fuliu (KI7) and Zigong (EX-CA1).

The patient was in a comfortable, supine position. After the skin was routinely disinfected and the acupoints were carefully localized, filiform $0.35 \mathrm{~mm} \times 40 \mathrm{~mm}$ sterilized, disposable needles (provided by Suzhou Hua Tuo Medical Instruments Co. Ltd, Suzhou, China) were inserted using the double hand-needle insertion technique. The depth of insertion was adjusted based on the patient's body size and the permissible depth of insertion of the specific acupoint. A technique using lifting, thrusting and twirling in a small range was performed until the appearance of De-qi, an obtained needle sensation, when there emerge a soreness, numbness and a feeling of distension around the point after the needle is inserted to a certain depth, and tenseness around the needle felt by the operator. Then, an even reinforcing-reducing technique was applied. The needle was retained for 40 minutes each time and manipulated twice during that time using a twirling technique in a small range.
The manipulation lasted 30 seconds for each acupoint. The acupuncture treatment consisted of two sessions each week for 12 consecutive weeks.

Auricular Acupressure. The selected auricular acupoints: sympathetic (AH6a), shenmen (TF4), adrenal gland (TG2p), subcortex (AT4), endocrine (CO18), kidney (CO10), heart (CO15) and liver (CO12).

After the above acupoints were sterilized with $75 \%$ alcohol, pieces of plaster with magnetic beads of proper size and good quality were stuck to the acupoints, which were then pressed slightly until the patient had an aching pain, numbness, distention and a warm sensation. The patients were asked to press the acupoints by themselves six times a day for a 3 minutes duration each time. It was explained that the strength of the pressing should make the local auricle congestive, flushed, hot and achy. The auricular acupressure was alternatively conducted on the two ears every 2 days. The plaster with magnetic beads was exchanged for a fresh set once a week.

The acupuncture process adhered to the Standards for Reporting Interventions in Controlled Trials of Acupuncture (STRICTA) criteria [24]. 
2.2.2. HRT Group. The patients were prescribed with oral Livial (Tibolone, made by Nanjing Oujianong Pharmaceutical Company Limited, Nanjing, China), in the dosage of one tablet a day $(2.5 \mathrm{mg} /$ tablet $)$ for 12 consecutive weeks.

One day before the treatment and at the end of the treatment, the levels of follicle stimulating hormone (FSH) and luteinizing hormone (LH) were measured with enzymelinked immunosorbent assay (ELISA) and the levels of $\mathrm{E}_{2}$ were measured with double antibiotic ELISA. All the reagents were provided by Lianxing Biological Technology Company, Tianjin, China.

2.3. Index and Method. The severity of hot flashes was defined as follows: mild-a fleeting, warm sensation without sweating or disruption of normal activities; moderate-a warm sensation associated with sweating, and disruption of normal activities; severe-a hot sensation associated with sweating and the discontinuation of normal activities $[16,19$, $23,25,26]$.

The score of the hot flash severity for a particular day is calculated by adding $1 \times$ the number of mild hot flashes +2 $x$ the number of moderate hot flashes $+3 \times$ the number of severe hot flashes $[16,19,23,25,26]$.

The frequency of hot flashes is the total number of the mild, moderate and severe hot flashes occurred during 24 hours.

2.4. Data Analysis. Results were analyzed by an independent university statistician using Statistical Package for Social Sciences (SPSS 13.0 for Windows), a computer software. Non-parametric Mann-Whitney tests were used to analyze the inter-group and intra-group differences of the severity and frequency of menopausal hot flashes. Analysis of variance (ANOVA) was used to compare the inter-group and intra-group differences of the serum levels of FSH, LH and $\mathrm{E}_{2}$. A $5 \%$ significance level $(P<.05)$ and two-tailed tests were used for all hypothesis tests. Ninety-five percent confidence intervals (CI) for the median differences between the acupuncture and auricular acupressure group and the HRT group were determined.

\section{Results}

3.1. The Baseline Characteristics. There were no significant difference between the two groups' baseline characteristics $(P>.05)$ (Table 1$)$.

3.2. The Severity of Hot Flashes. There was no significant difference between the two groups' severity of hot flashes before treatment. After the 12 weeks of treatment, both groups' severity of hot flashes decreased significantly $(P<$ $.05)$ with no significant difference between the groups $(P>$ $.05)$. The 4 -week follow-up showed that both groups were alleviated significantly when compared with pre-treatment $(P<.05)$ and HRT had greater effects $(P<.05)$ (Table 2$)$.

3.3. The Frequency of Hot Flashes. There was no significant difference between the two groups before treatment. After the 12 weeks of treatment, the frequency of the menopausal hot flashes in both groups were reduced significantly $(P<$ $.05)$ and HRT had greater effects $(P<.05)$ (Table 2$)$. The 4 week follow-up showed that the frequency of hot flashes in the two groups both decreased significantly when compared with pre-treatment $(P>.05)$ and the patients in the HRT group were alleviated more $(P<.05)$ (Table 2$)$.

3.4. The Serum Levels of FSH, LH and $E_{2}$. Before treatment, there was no significant difference in the serum levels of $\mathrm{FSH}, \mathrm{LH}$ and $\mathrm{E}_{2}$ between the acupuncture and auricular acupressure group and the HRT group. After treatment, the levels of FSH decreased significantly and the levels of $\mathrm{E}_{2}$ increased significantly in both of the two groups and the levels of LH decreased significantly in the HRT group $(P<$ .05) (Table 2). The serum levels of FSH, LH and $\mathrm{E}_{2}$ in the HRT group changed more $(P<.05)$ (Table 2$)$.

3.5. Adverse Result. No side-effect was reported in either group during the period of the research or in the follow-up phase.

\section{Discussion}

Although menopause is associated with changes in the hypothalamic and pituitary hormones that regulate the menstrual cycle, menopause is not a central event, but rather primary ovarian failure [27]. As the hypothalamicpituitary-ovarian axis remains intact during the menopausal transition, FSH levels rise in response to ovarian failure and the absence of negative feedback from the ovary [27]. Atresia of the follicular apparatus, in particular the granulosa cells, results in the reduced production of estrogen and inhibin, which leads to the reduced inhibin levels and the elevated FSH levels, a cardinal sign of menopause [27]. Correlations between endocrine levels and symptom severity ratings over time revealed that hot flash severity was significantly and positively related to FSH [28]. Investigations of hormonal connections between hot flash severity and reproductive hormones in Study of Women's Health Across the Nation (SWAN), Melbourne Midlife Women's Health Project (MMWHP) and Penn Ovarian Aging Study cohorts found that decreased serum $\mathrm{E}_{2}$ and increased serum FSH were associated with the increases in hot flash severity [2931]. In an analysis of SWAN data, which modeled the effects of $\mathrm{FSH}$ and $\mathrm{E}_{2}$ (and other reproductive hormones) together, Randolph and colleagues noted that FSH was associated with hot flash prevalence and frequency [7].

It is well known that acupuncture is associated with homeostatic regulation, and possess effects such as buffering hormonal disturbance, modulating ovulation, as well as improving psychological or behavioral abnormity [3234]. Acupuncture in specific acupoints has been found to significantly increase blood concentrations of $E_{2}$ in the ovariectomized rats [35], while reducing the elevated plasma LH due to ovariectomy [36]; in addition, acupuncture also restored the number of gonadotropin-releasing hormone $(\mathrm{GnRH})$ neurons in the ovariectomized rats [35]. In another 
TABLE 2: The severity and frequency of hot flashes and the serum levels of FSH, $\mathrm{LH}$ and $\mathrm{E}_{2}$.

\begin{tabular}{|c|c|c|}
\hline Item & Acupuncture and auricular acupressure group $(n=19)$ & HRT group $(n=24)$ \\
\hline \multicolumn{3}{|l|}{ Hot flash severity } \\
\hline Pre-treatment & $14.71 \pm 3.81(95 \%$ CI $12.96-16.52)$ & $15.28 \pm 4.06(95 \%$ CI $13.53-16.96)$ \\
\hline Post-treatment & $3.86 \pm 0.84^{*}(95 \%$ CI $3.40-4.45)$ & $3.71 \pm 0.65 *(95 \%$ CI $3.44-3.97)$ \\
\hline Follow-up & $4.45 \pm 0.90^{*, \#}(95 \%$ CI 3.92-4.95) & $3.25 \pm 0.34 *(95 \%$ CI $3.00-3.33)$ \\
\hline \multicolumn{3}{|l|}{ Hot flash frequency } \\
\hline Pre-treatment & $14.21 \pm 2.42(95 \%$ CI $13.37-15.88)$ & $15.11 \pm 4.96(95 \%$ CI $13.04-17.30)$ \\
\hline Post-treatment & $10.32 \pm 3.13^{*, \#}(95 \%$ CI $8.80-12.32)$ & $7.48 \pm 2.69 *(95 \%$ CI $6.33-8.59)$ \\
\hline Follow-up & $7.69 \pm 1.48^{*, \#}(95 \%$ CI 7.12-8.63) & $5.58 \pm 1.64 *(95 \%$ CI $4.86-6.22)$ \\
\hline \multicolumn{3}{|l|}{ FSH (mIU/mL) } \\
\hline Pre-treatment & $51.5 \pm 13.9(95 \%$ CI 46.7-58.7) & 53.0. $\pm 14.3(95 \%$ CI 46.9-59.0) \\
\hline Post-treatment & $40.4 \pm 9.6^{*, \#}(95 \%$ CI $34.6-44.5)$ & $22.7 \pm 5.1^{*}(95 \%$ CI $20.6-24.8)$ \\
\hline \multicolumn{3}{|l|}{$\mathrm{LH}(\mathrm{mIU} / \mathrm{mL})$} \\
\hline Pre-treatment & $33.7 \pm 6.4(95 \%$ CI 30.2-37.0) & $32.9 \pm 7.1(95 \%$ CI 29.9-35.9) \\
\hline Post-treatment & $31.9 \pm 5.7^{\#}(95 \%$ CI $28.6-34.9)$ & $15.6 \pm 4.8^{*}(95 \%$ CI $13.6-17.6)$ \\
\hline \multicolumn{3}{|l|}{$\mathrm{E}_{2}(\mathrm{pg} / \mathrm{mL})$} \\
\hline Pre-treatment & $37.1 \pm 8.9(95 \%$ CI $32.0-41.5)$ & $39.4 \pm 7.0(95 \%$ CI $36.4-42.3)$ \\
\hline Post-treatment & $45.4 \pm 7.3^{*, \#}(95 \%$ CI $41.7-49.4)$ & $71.5 \pm 13.4^{*}(95 \%$ CI $65.8-77.1)$ \\
\hline
\end{tabular}

FSH, follicle stimulating hormone; LH, luteinizing hormone; $\mathrm{E}_{2}$, estradiol; CI, Confidence intervals.

${ }^{*} P<.05$, compared with pre-treatment in the same group.

${ }^{\#} P<.05$, compared with the HRT group.

study, acupuncture was found to improve the reproductive disorders induced by ovariectomy in rats through modulating the blood $\mathrm{E}_{2}$ levels [37]. Acupuncture may improve the function of the hypothalamic-pituitary-ovarian axis, increase blood adrenogenous androgen level and facilitate its transformation into estrogen by aromatic enzyme in the brain, liver and fat tissues [38-40].

The present study showed that acupuncture and auricular acupressure significantly relieve the severity and frequency of menopausal hot flashes. The levels of FSH decreased significantly and the level of $\mathrm{E}_{2}$ increased significantly in both of the two groups after treatment. As the increased levels of FSH and the lowered level of $\mathrm{E}_{2}$ are mainly associated with hot flashes during the menopausal transition [29-31], it may be partly through decreasing the levels of FSH and increasing the levels of $\mathrm{E}_{2}$ that acupuncture and auricular acupressure alleviate the severity and frequency of menopausal hot flashes of the bilaterally ovariectomized Chinese women. In comparison with HRT, although acupuncture did not change hormone levels as significantly as HRT in this study, the bilaterally ovariectomized women's own functions may be regulated with the use of acupuncture, while HRT restores the body's hormone level by exogenous hormones.

The protocol of the acupuncture treatments comes from a combination of literatures and clinical experiences. There is no corresponding name to menopausal hot flashes in ancient books of traditional Chinese medicine (TCM). Based on Zang-fu organs in TCM, disorders of the kidney and liver are generally considered as the main pathogenesis. According to the principle of reinforcing the kidney and regulating the liver, the main acupoints selected were Sanyinjiao (SP6), Fengchi (GB20), Hegu (LI4), Quchi (LI11), Guanyuan
(CV4), Dazhui (GV14), Fuliu (KI7) and Zigong (EX-CA1). Among them, Sanyinjiao (SP6), Fengchi (GB20), Hegu (LI4) and Quchi (LI11) have been selected as the main acupoints in most of the clinical researches on menopausal hot flashes $[19,23]$. Sanyinjiao (SP6) is an important acupoint to treat disorders of the spleen, liver and kidney [41]. It is able to soften and harmonize the Liver and to benefit the kidney Qi, based on the ancient classics of acupuncture [42]. Fengchi (GB20) works to regulate the function of liver and remove heat from the head and eye, based on the ancient acupuncture records and the Meridian Theory of TCM [41]. Hegu (LI4) functions to clear heat in the Upper and Middle Jiao [41]. Quchi (LI11) has been acknowledged to produce hypothermia in normal subject [42]. Guanyuan (CV4) is an important acupoint on the Conception Vessel and functions to reinforce kidney Qi and replenish Qi and blood [41]. Sanyinjiao (SP6) and Guanyuan (CV4) have been found to increase the gonadal hormone levels [43]. Dazhui (GV14) has been found to produce hypothermia in normal adult [44]. Fuliu (KI7) can regulate kidney Qi and sweating [41]. Zigong (EX-CA1) is an important empirical one for treating female disorders [41].

According to the theory of TCM, all channels of the body and all 12 meridians are closely connected with the ear [45]. The earliest record of ear acupuncture is in Huangdi Neijing (The Yellow Emperor's Classic of Internal Medicine). In the famous Chinese medical classics, a great number of acupuncture treatments were summarized, among which, there are the specific acupuncture points on the external ear for relief of certain disorders. Auricular acupressure could associate the meridians of the body, regulate Qi and activate blood, regulate Zang-fu organs and promote good metabolism according to the Meridian Theory of TCM [45]. 
There are 200 acupoints on the outer ear. Auricular acupressure's effectiveness and non-invasiveness make it easily accepted by both patients and doctors [45, 46]. Auricular acupressure works by stimulating the central nervous system through the cranial and spinal nerves on the auricle of the ear. This stimulation results in increasing neurotransmitters within the pituitary and spinal cord of the central nervous system [45]. All the auricular acupoints selected in the study were associated with enforcing kidney Qi and regulating liver Qi.

Acupuncture has been found to significantly reduce the severity of nocturnal hot flashes in post-menopausal women [19]. Standardized and individually tailored acupuncture treatment was also found to significantly decrease the severity of hot flashes in symptomatic post-menopausal women when compared with placebo acupuncture of equal duration [23]. Another study showed that acupuncture and applied relaxation significantly reduced the number of menopausal hot flashes [22]. The present study showed that acupuncture and auricular acupressure significantly relieve the severity and frequency of menopausal hot flashes. However, as the sample size of the present study was small and the shamacupuncture was not used, the conclusion may be somewhat limited.

Although the double-blind randomized controlled trial (RCT) has been known as the gold standard in clinical researches, the biggest challenges and the difficulties to the researchers in the fields of clinical acupuncture and moxibustion are the design of an ideal placebo-control method and the credibility and ethics behind sham acupuncture [4752]. An ideal acupuncture placebo that avoids the necessity of penetration of the skin and shows the same psychological impact has not yet been found. A study has shown that for non-drug interventions including acupuncture, it was difficult to establish a placebo or sham control that is both inert and indistinguishable [53]. It concluded that although randomized trials investigating the specific effects of acupuncture have used a great variety of sham interventions as controls, the sham interventions as "placebo" controls seem misleading and scientifically unacceptable [53]. Another study demonstrated that the control interventions were equally as effective as acupuncture in alleviating pain in conditions that are predominantly associated with affective components such as migraines or lower back pain, but not those with a more pronounced sensory component, such as osteoarthritis of the knee or lateral epicondylalgia [54]. Some previous research also showed that nearly $40 \%$ of the participants in clinical research were able to detect a difference between the active and placebo needles at active points [55]. In a clinical study on the effect of acupuncture in treating post-menopausal hot flashes, although they combined the use of placebo needles with sham points in the placebo treatment, a difference in the patients' expectations of benefit was still found, and they considered finding an optimal placebo for acupuncture remained a challenge for future studies [23]. In addition, as most of the middle-aged Chinese people have had experiences receiving acupuncture treatments, it is even more difficult to simulate real acupuncture in clinical researches. This is why the study did not attempt to use sham-acupuncture as a control. Although this may introduce bias into trials, extensive details regarding the baseline characteristics of the recruited women were collected before treatment and between the two groups, no significant difference in the patients' detailed baseline characteristics existed. Further research with larger samples needs to be conducted.

\section{Conclusions}

Acupuncture and auricular acupressure can be used as alternative treatments to relieve menopausal hot flashes for those bilaterally ovariectomized women who are unable or unwilling to receive HRT.

\section{Funding}

Natural Medicine Research UK (to J.Z.); China Postdoctoral Science Foundation (no. 20080441265 to J.Z.); Zhejiang Traditional Chinese Medicine Foundation (no. 2008YA015 to J.Z.); Zhejiang Province Postdoctoral Science Foundation (to J.Z.); China Postdoctoral Science Foundation (no. 20070421188 to F.Q.); Outstanding Young Medical Scientist Foundation of Zhejiang Province (no. 2008QN022); Zhejiang Traditional Chinese Medicine Foundation (no. 2008YB010).

\section{Acknowledgemts}

The authors wish to thank the patients included in the research. The authors would also like to express their deep thanks to Elizabeth Burrows from Global College of Long Island University, Brooklyn, NY, USA for her kind editing. J. Zhou and F. Qu contributed equally to this work.

\section{References}

[1] R. R. Freedman, "Physiology of hot flashes," American Journal of Human Biology, vol. 13, no. 4, pp. 453-464, 2001.

[2] F. Kronenberg, "Hot flashes: epidemiology and physiology," Annals of the New York Academy of Sciences, vol. 592, pp. 52$86,1990$.

[3] C. B. Hammond, "Climacteric," in Danforth's Obstetrics and Gynecology, J. R. Scott, P. J. DiSaia, C. B. Hammond, and W. N. Spellacy, Eds., pp. 771-790, J. B. Lippincott, Philadelphia, Pa, USA, 1994.

[4] F. Kronenberg, "Hot flashes: phenomenology, quality of life, and search for treatment options," Experimental Gerontology, vol. 29, no. 3-4, pp. 319-336, 1994.

[5] K. D. Juang, S. J. Wang, S. R. Lu, S. J. Lee, and J. L. Fuh, "Hot flashes are associated with psychological symptoms of anxiety and depression in periand post- but not pre-menopausal women," Maturitas, vol. 52, pp. 119-126, 2005.

[6] K. Visvanathan, L. Gallicchio, C. Schilling et al., "Cytochrome gene polymorphisms, serum estrogens, and hot flushes in midlife women," Obstetrics and Gynecology, vol. 106, no. 6, pp. 1372-1381, 2005.

[7] J. F. Randolph Jr., M. F. Sowers, I. Bondarenko et al., "The relationship of longitudinal change in reproductive hormones and vasomotor symptoms during the menopausal transition," 
Journal of Clinical Endocrinology and Metabolism, vol. 90, no. 11, pp. 6106-6112, 2005.

[8] B. Thompson, S. A. Hart, and D. Durno, "Menopausal age and symptomatology in a general practice," Journal of Biosocial Science, vol. 5, no. 1, pp. 71-82, 1973.

[9] I. V. Tataryn, P. Lomax, and J. G. Bajorek, "Postmenopausal hot flushes: a disorder of thermoregulation," Maturitas, vol. 2, no. 2, pp. 101-107, 1980.

[10] V. Stearns, K. L. Beebe, M. Iyengar, and E. Dube, "Paroxetine controlled release in the treatment of menopausal fot flashes: a randomized controlled trial," Journal of the American Medical Association, vol. 289, no. 21, pp. 2827-2834, 2003.

[11] Writing Group for the Women's Health Initiative Investigators, "Risks and benefits of estrogen plus progestin in healthy postmenopausal women: principal results from the women's health initiative randomized controlled trial," Journal of the American Medical Association, vol. 288, pp. 321-33, 2002.

[12] M. T. Connelly, M. Richardson, and R. Platt, "Prevalence and duration of postmenopausal hormone replacement therapy use in a managed care organization," Journal of General Internal Medicine, vol. 15, no. 8, pp. 542-550, 2000.

[13] D. A. Hill, N. S. Weiss, and A. Z. LaCroix, "Adherence to postmenopausal hormone therapy during the year after the initial prescription: a population based study," American Journal of Obstetrics \& Gynecology, vol. 182, pp. 270-276, 2000.

[14] S. P. Yang, L. F. He, and J. Yu, "Levels of hypothalamic proopimelanocortin mRNA and estrogen binding sites during preovulatory GnRH surge in rat," Acta Zoologica Sinica, vol. 33, p. 73, 1998.

[15] Y Wyon, R Lindgren, T Lundeberg, and M Hammar, "Effects of acupuncture on climacteric vasomotor symptoms, quality of life and urinary excretion of neuropeptides among postmenopausal women," Menopause, vol. 2, pp. 3-12, 1995.

[16] A. Vincent, D. L. Barton, J. N. Mandrekar et al., "Acupuncture for hot flashes: a randomized, sham-controlled clinical study," Menopause, vol. 14, no. 1, pp. 45-52, 2007.

[17] Y. Wyon, K. Wijma, E. Nedstrand, and M. Hammar, "A comparison of acupuncture and oral estradiol treatment of vasomotor symptoms in postmenopausal women," Climacteric, vol. 7, no. 2, pp. 153-164, 2004.

[18] H. Dong, F. Lüdicke, I. Comte, A. Campana, P. Graff, and P. Bischof, "An exploratory pilot study of acupuncture on the quality of life and reproductive hormone secretion in menopausal women," Journal of Alternative and Complementary Medicine, vol. 7, no. 6, pp. 651-658, 2001.

[19] M. I. Huang, Y. Nir, B. Chen, R. Schnyer, and R. Manber, "A randomized controlled pilot study of acupuncture for postmenopausal hot flashes: effect on nocturnal hot flashes and sleep quality," Fertility and Sterility, vol. 86, pp. 700-710, 2006.

[20] F. Xie, C.-F. Wu, W.-P. Lai et al., "The osteoprotective effect of Herba epimedii (HEP) extract in vivo and in vitro," EvidenceBased Complementary and Alternative Medicine, vol. 2, no. 3, pp. 353-361, 2005.

[21] S. Tsukamoto, M. Aburatani, and T. Ohta, "Isolation of CYP3A4 inhibitors from the black cohosh (Cimicifuga racemosa)," Evidence-Based Complementary and Alternative Medicine, vol. 2, no. 2, pp. 223-226, 2005.

[22] E. Zaborowska, J. Brynhildsen, S. Damberg et al., "Effects of acupuncture, applied relaxation, estrogens and placebo on hot flushes in postmenopausal women: an analysis of two prospective, parallel, randomized studies," Climacteric, vol. 10, no. 1, pp. 38-45, 2007.
[23] Y. Nir, M. I. Huang, R. Schnyer, B. Chen, and R. Manber, "Acupuncture for postmenopausal hot flashes," Maturitas, vol. 56, no. 4, pp. 383-395, 2007.

[24] H. MacPherson, A. White, M. Cummings, K. A. Jobst, K. Rose, and R. C. Niemtzow, "Standards for reporting interventions in controlled trials of acupuncture: the STRICTA recommendations," Journal of Alternative and Complementary Medicine, vol. 8, no. 1, pp. 85-89, 2002.

[25] W. H. Utian, D. Shoupe, G. Bachmann, J. V. Pinkerton, and J. H. Pickar, "Relief of vasomotor symptoms and vaginal atrophy with lower doses of conjugated equine estrogens and medroxyprogesterone acetate," Fertility and Sterility, vol. 75, no. 6, pp. 1065-1079, 2001.

[26] J. A. Sloan, C. L. Loprinzi, P. J. Novotny, D. L. Barton, B. I. Lavasseur, and H. Windschitl, "Methodologic lessons learned from hot flash studies," The Journal of Clinical Oncology, vol. 19, no. 23, pp. 4280-4290, 2001.

[27] J. S. Berek, Berek \& Novak's Gynecology, Lippincott Williams \& Wilkins, Philadelphia, Pa, USA, 2007.

[28] N. F. Woods, K. Smith-Dijulio, D. B. Percival, E. Y. Tao, H. J. Taylor, and E. S. Mitchell, "Symptoms during the menopausal transition and early postmenopause and their relation to endocrine levels over time: observations from the Seattle Midlife Women's Health Study," Journal of Women's Health, vol. 16, pp. 667-677, 2007.

[29] E. W. Freeman, M. D. Sammel, H. Lin, C. R. Gracia, S. Kapoor, and T. Ferdousi, "The role of anxiety and hormonal changes in menopausal hot flashes," Menopause, vol. 12, no. 3, pp. 258266, 2005.

[30] E. B. Gold, G. Block, S. Crawford et al., "Lifestyle and demographic factors in relation to vasomotor symptoms: baseline results from the study of women's health across the nation," American Journal of Epidemiology, vol. 159, no. 12, pp. 1189-1199, 2004.

[31] J. R. Guthrie, L. Dennerstein, J. R. Taffe, P. Lehert, and H. G. Burger, "Hot flushes during the menopause transition: a longitudinal study in Australian-born women," Menopause, vol. 12, no. 4, pp. 460-467, 2005.

[32] J. V. Clement, L. Mcloughlin, P. J. Lowry, G. M. Besser, L. H. Rees, and H. L. Wen, "Acupuncture in heroin addicts: changes in Met-enkephalin and beta-endorphin in blood and cerebrospinal liquid," The Lancet, vol. 2, pp. 380-383, 1979.

[33] E. H. Kim, Y. J. Kim, H. J. Lee et al., "Acupuncture increases cell proliferation in dentate gyrus after transient global ischemia in gerbils," Neuroscience Letters, vol. 297, pp. 21-24, 2001.

[34] E. Stener-Victorin, U. Waldenström, U. Tägnfors, T. Lundeberg, G. Lindstedt, and P. O. Janson, "Effects of electroacupuncture on anovulation in women with polycystic ovary syndrome," Acta Obstetricia et Gynecologica Scandinavica, vol. 79, no. 3, pp. 180-188, 2000.

[35] H. Zhao, Z. Z. Tian, and B. Y. Chen, "An important role of corticotropin-releasing hormone in electroacupuncture normalizing the subnormal function of hypothalamus-pituitaryovary axis in ovariectomized rats," Neuroscience Letters, vol. 349, pp. 25-28, 2003.

[36] B.-Y. Chen, "Acupuncture normalizes dysfunction of hypothalamic-pituitary-ovarian axis," Acupuncture and Electro-Therapeutics Research, vol. 22, no. 2, pp. 97-108, 1997.

[37] X. Yao, X. Q. Wang, S. L. Ma, and B. Y. Chen, "Electroacupuncture stimulates the expression of prolactin-releasing peptide (PrRP) in the medulla oblongata of ovariectomized rats," Neuroscience Letters, vol. 411, pp. 243-248, 2007.

[38] J. H. Lin, S. H. Liu, W. W. Chan, L. S. Wu, and W. P. Pi, "Effects of electroacupuncture and gonadotropin-releasing hormone 
treatments on hormone changes in anoestrous sows," The American Journal of Chinese Medicine, vol. 16, pp. 117-126, 1988.

[39] T. Aso, T. Motohashi, M. Murata, T. Nishimura, and K. Kakizaki, "The influence of acupuncture stimulation on plasma levels of $\mathrm{LH}, \mathrm{FSH}$, progesterone and estradiol in normally ovulating women," The American Journal of Chinese Medicine, vol. 4, pp. 391-401, 1976.

[40] J. Yu, H. M. Zheng, and S. M. Ping, "Changes in serum FSH, LH and ovarian follicular growth during electroacupuncture for induction of ovulation," Chung Hsi I Chieh Ho Tsa Chih, vol. 9, pp. 199-202, 1989.

[41] L. X. Huang, Series of Ancient Classics on Acu-Moxibustion Science, Hua-xia Press, Beijing, China, 1997.

[42] M. T. Lin, A. Chandra, S. M. Chen-Yen, and Y. F. Chern, "Needle stimulation of acupuncture loci Chu-Chih (LI-11) and $\mathrm{Ho}-\mathrm{Ku}$ (LI-4) induces hypothermia effects and analgesia in normal adults," The American Journal of Chinese Medicine, vol. 9, pp. 74-83, 1981.

[43] H. L. Wang, L. Hu, and X. Z. Gao, "The effects of Sanyinjiao (SP-6) and Guanyuan (CV4) in increasing the gonadal hormone levels of menopausal model rats," Zhen Ci Yan Jiu, vol. 28, pp. 124-127, 2003.

[44] M. T. Lin, G. G. Liu, J. J. Soong, Y. F. Chern, and K. M. Wu, "Effects of stimulation of acupuncture loci TaChuei (Go-14), Nei-Kuan (EH-6) and Tsu-San-Li (St-36) on thermoregulatory function of normal adults," The American Journal of Chinese Medicine, vol. 7, pp. 324-332, 1979.

[45] Z. X. Guan, Chinese Auricular Acupuncture, Shanghai Press of Science and Technology, Shanghai, China, 1995.

[46] T. I. Usichenko, M. Hermsen, T. Witstruck et al., "Auricular acupuncture for pain relief after ambulatory knee arthroscopy-a pilot study," Evidence-Based Complementary and Alternative Medicine, vol. 2, no. 2, pp. 185-189, 2005.

[47] J.-L. Tang, S.-Y. Zhan, and E. Ernst, "Review of randomised controlled trials of traditional Chinese medicine," British Medical Journal, vol. 318, no. 7203, pp. 160-161, 1999.

[48] C. Vincent and G. Lewith, "Placebo controls for acupuncture studies," Journal of the Royal Society of Medicine, vol. 88, no. 4, pp. 199-202, 1995.

[49] K. J. Sherman, C. J. Hogeboom, D. C. Cherkin, and R. A. Deyo, "Description and validation of a noninvasive placebo acupuncture procedure," Journal of Alternative and Complementary Medicine, vol. 8, no. 1, pp. 11-19, 2002.

[50] M. Arthur, A. Killy, and D. K. Herbert, "Investigating alternative medicine therapies in randomized controlled trials," Journal Of the American Medical Association, vol. 280, pp. 1626-1628, 1998.

[51] K. Linde and F. Dincer, "How informed is consent in shamcontrolled trials of acupuncture?" Journal of Alternative and Complementary Medicine, vol. 10, no. 2, pp. 379-385, 2004.

[52] E. J. Emanuel and F. G. Miller, "The ethics of placebocontrolled trials—a middle ground," The New England Journal of Medicine, vol. 345, no. 12, pp. 915-919, 2001.

[53] F. Dincer and K. Linde, "Sham interventions in randomized clinical trials of acupuncture-a review," Complementary Therapies in Medicine, vol. 11, no. 4, pp. 235-242, 2003.

[54] I. Lund and T. Lundeberg, "Are minimal, superficial or sham acupuncture procedures acceptable as inert placebo controls?" Acupuncture in Medicine, vol. 24, no. 1, pp. 13-15, 2006.

[55] P. White, G. Lewith, V. Hopwood, and P. Prescott, "The placebo needle, is it a valid and convincing placebo for use in acupuncture trials? A randomised, single-blind, cross-over pilot trial," Pain, vol. 106, no. 3, pp. 401-409, 2003. 


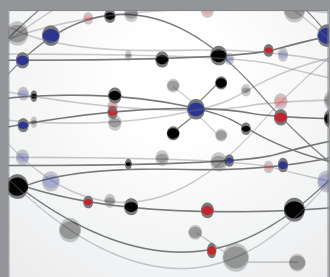

The Scientific World Journal
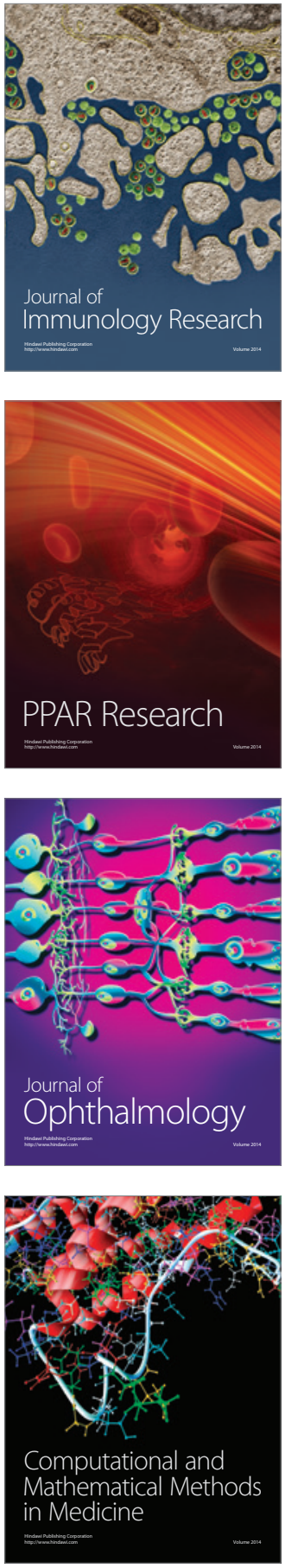

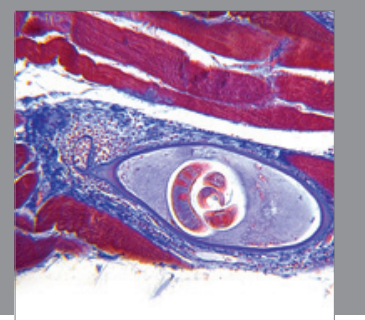

Gastroenterology

Research and Practice
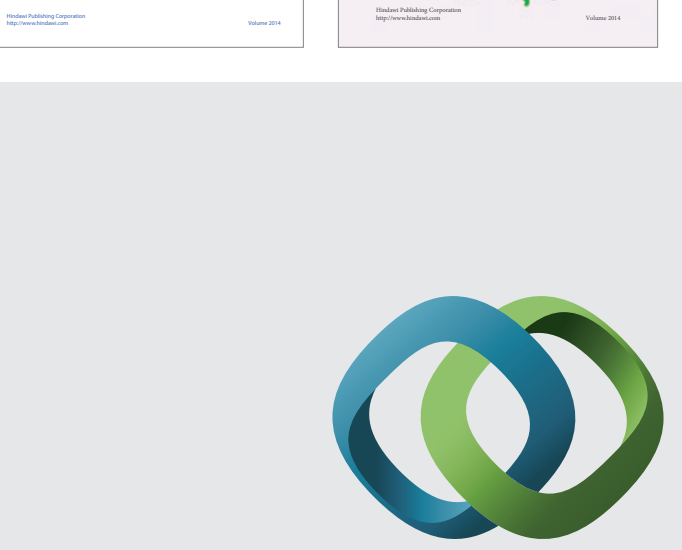

\section{Hindawi}

Submit your manuscripts at

http://www.hindawi.com
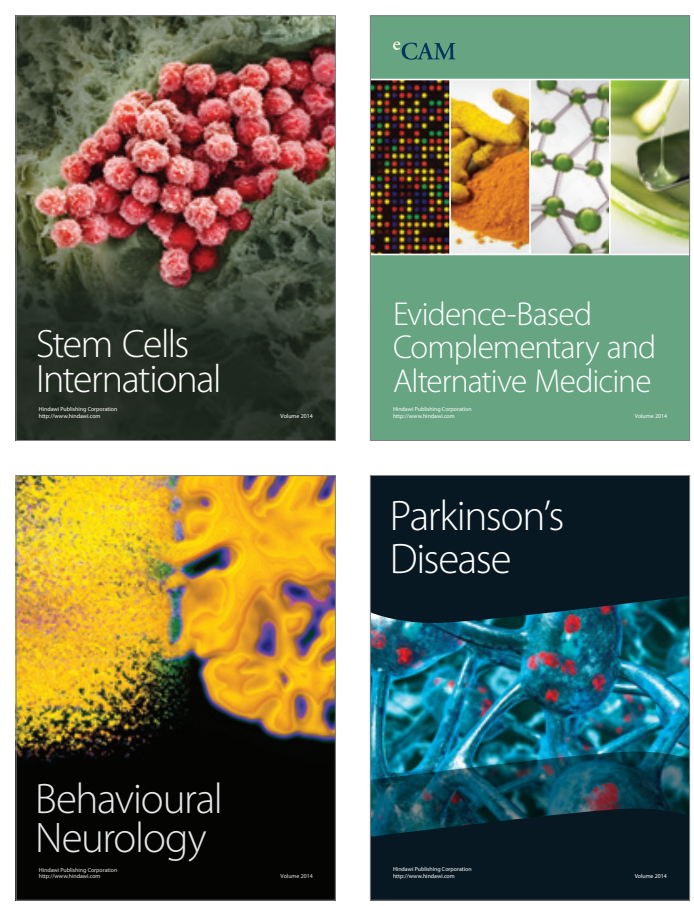

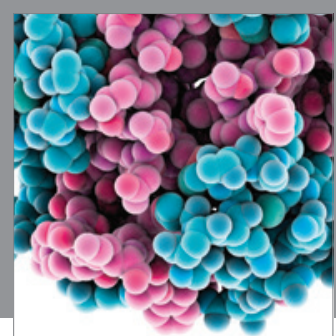

Journal of
Diabetes Research

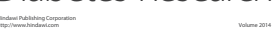

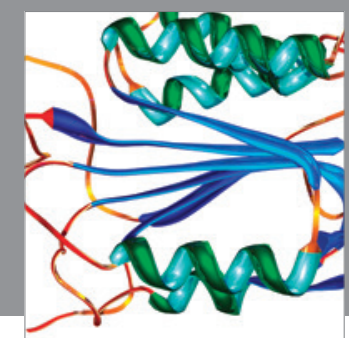

Disease Markers
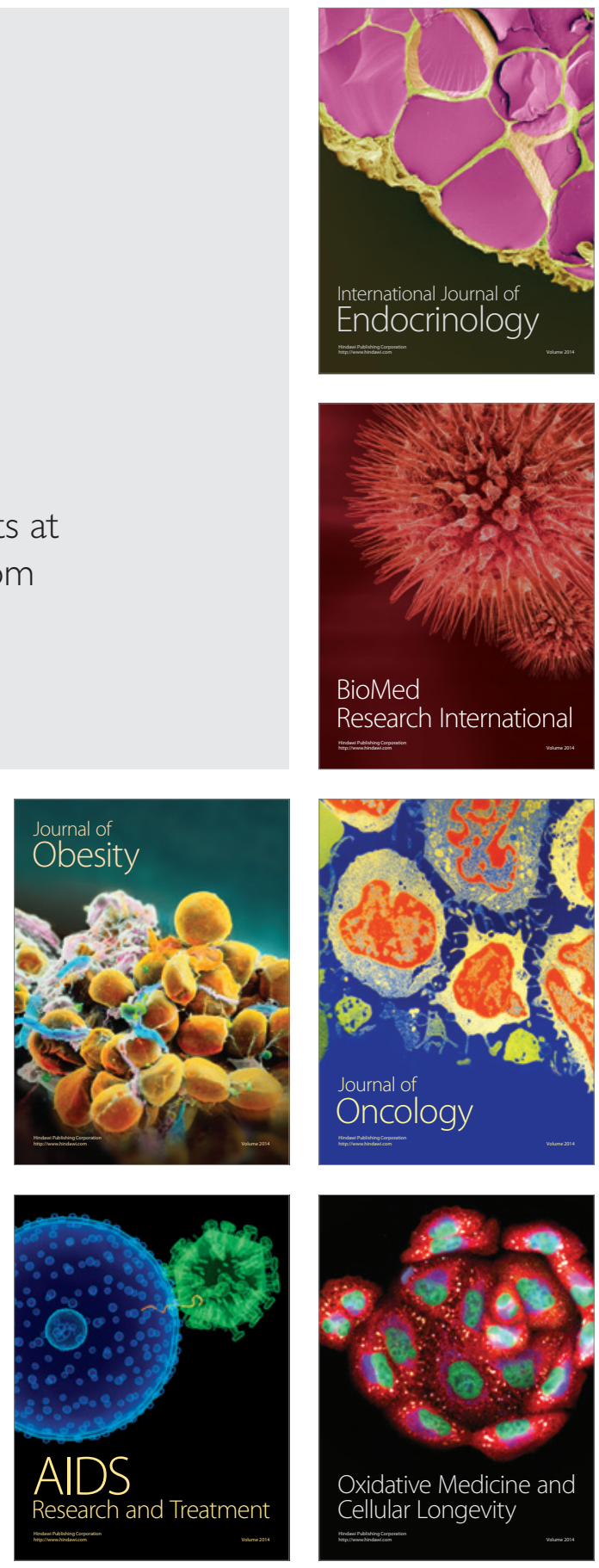